\title{
$\infty \sqrt{1}$ Stroke and \\ Who may benefit from lower dosages of intravenous tissue plasminogen activator? Results from a cluster data analysis
}

To cite: Dong $\mathrm{Y}$, Han $\mathrm{Y}$, Shen $\mathrm{H}$, et al. Who may benefit from lower dosages of intravenous tissue plasminogen activator? Results from a cluster data analysis. Stroke \& Vascular Neurology 2020;5: e000388. doi:10.1136/svn-2020-000388

- Additional material is published online only. To view please visit the journal online (http://dx.doi.org/10.1136/svn2020-000388)

YLW and QD contributed equally.

YD and YH are joint first authors.

Received 25 March 2020

Revised 26 May 2020

Accepted 28 May 2020

Published Online First

1 July 2020

Check for updates

(c) Author(s) (or their employer(s)) 2020. Re-use permitted under CC BY-NC. No commercial re-use. See rights and permissions. Published by BMJ.

For numbered affiliations see end of article.

Correspondence to Dr Qiang Dong;

dong_qiang@fudan.edu.cn

Dr Yilong Wang;

yilong528@aliyun.com

\section{ABSTRACT}

Background The risk of symptomatic intracranial haemorrhage (sICH) after thrombolysis is low but severe. Lower dose of alteplase may reduce the risk of $\mathrm{sICH}$. We aim to identify subsets of patients who could benefit from lower dose of alteplase compared with standard dose. Methods Data from two observational registries were pooled together. A total of 3479 patients who had acute ischaemic stroke were entered into the interaction tree model. The response variable was the rate of $\mathrm{SICH}$ per the definition of the National Institute of Neurological Disorders and Stroke Study. Clinical improvement was measured by the National Institutes of Health Stroke Scale (NIHSS) and defined as NIHSS 0 or 1 or an improvement of more than 4 points (within 7 days or at discharge). Rare event logistic regression was performed to analyse the $\mathrm{OR}$ of safety outcome.

Results To optimise the interaction effect between tissue plasminogen activator (tPA) dosage (standard/lower) and patient subgroups, three subgroups based on the severity of stroke were identified: (1) NIHSS $\leq 4$, (2) NIHSS between 5 and 14 , and (3) NIHSS $\geq 15$. The estimated difference of OR of having sICH was $2.71(95 \% \mathrm{Cl} 0.80$ to $7.69, \mathrm{p}=0.10)$ for mild, $0.13(95 \% \mathrm{Cl} 0.02$ to $0.68, p=0.01)$ for moderate, and $0.65(95 \% \mathrm{Cl} 0.19$ to $2.55, \mathrm{p}=0.52)$ for severe, respectively. In addition, patients who had moderate stroke treated with lower dose had comparable efficacy outcome (OR 1.23, 95\% $\mathrm{Cl} 0.71$ to $2.13, \mathrm{p}=0.45$ ).

Conclusion Our analysis demonstrated that in patients who had moderate stroke, lower doses of alteplase are associated with significant sICH reduction and non-inferior performance in efficacy, compared with those in the standard dose group.

Trial registration number The TIMS-China was a national prospective stroke registry on thrombolytic therapy using intravenous tPA in patients who had acute ischaemic stroke. The results were initially published in 2012 without a clinical trial registration number. The Shanghai Stroke Service System was registered at www.clinicaltrial.gov (NCT02735226).

\section{INTRODUCTION}

Although intravenous tissue plasminogen activator (tPA) is the standard treatment for patients who had acute ischaemic stroke (AIS), lower dosage of tPA has not been well studied until the completion of the Enhanced Control of Hypertension and Thrombolysis Stroke Study (ENCHANTED). ${ }^{1}$ In the earlier pilot studies of AIS, ${ }^{23}$ limited dose tiers were explored. Since the National Institute of Neurological Disorders and Stroke Study (NINDS) was published, there have been many debates on the appropriate dosages of intravenous tPA, ${ }^{4}$ especially for AIS in Asian populations. ${ }^{5-10}$

Several subgroup studies have focused on the prediction models for symptomatic intracranial haemorrhage (sICH) or poor outcome related to intravenous tPA. ${ }^{11-15}$ However, there is a clinically relevant net positive effect of intravenous tPA in treating patients with AIS that outweighs the risk of sICH. ${ }^{14}$ This finding also applies to those treated with lower doses of tPA. ${ }^{1-3} 16$ On the other hand, in the subset of patients who had ischaemic stroke with high risk of bleeding, there is limited evidence on whether they could benefit from a lower dose of intravenous tPA. Furthermore, low-dose intravenous tPA may have its applications for the following reasons: more affordable in developing countries and possibly a safer option for elderly patients with AIS. $^{17}$

In the present study, cluster analysis was used to identify those who could benefit from lower doses of intravenous tPA. Cluster analysis uses algorithms to group a patient population in a data-driven manner, without any predesigned definitions. It is different from the subgroup analysis of large trials, ${ }^{1}$ where populations are predefined and statistical testing is used to identify differences. Our cluster analysis was conducted using the data pooled from two observational registries that studied differences in the rate of sICH and clinical outcome in patients who had a stroke treated with either standard or lower doses of intravenous tPA. The objective of this cluster analysis was to identify patients who could benefit the most from lower 
doses of intravenous tPA, but with lower rate of sICH and similar efficacy in outcome compared with those treated with standard dose.

\section{METHODS}

\section{Clinical study design and subjects}

Data from two observational registries were pooled. The Thrombolysis Implementation and Monitor of Acute Ischemic Stroke in China (TIMS-China) study was a national prospective stroke registry of thrombolytic therapy with intravenous tPA in patients with AIS. ${ }^{6}$ Started in May 2007, TIMS-China included 167 centres and 1109 patients who were treated with intravenous tPA within 4.5 hours of onset. The Shanghai Stroke Service System (4S) was designed as an ongoing prospective regional stroke network registry. ${ }^{18}$ In 2013, the 4 S registry was initiated to include 11 regional stroke centres in Shanghai with a web-based database using automatic extraction from the electronic medical record systems. ${ }^{17}$ The $4 \mathrm{~S}$ database has enrolled 2370 patients who were treated with intravenous tPA by 30 June 2014 . The ethical reviews were approved and declared in both these registries. ${ }^{6} 18$

All participants in the TIMS-China and participants treated with intravenous tPA in the 4S during 2013-2014 were included in this analysis. All inclusion and exclusion criteria have been published previously. ${ }^{6} 18$ The characteristics of the patients in the two databases are listed in online supplementary table 1. Patients in TIMS-China suffered from more severe strokes and had more history of stroke, who were also more likely to have been treated with lower doses of tPA. In 4S, more patients were female, had milder stroke and had more history of diabetes. Due to the heterogeneity and limited sample size of patients treated with lower doses of intravenous IPA, the two databases were pooled together in order to achieve a better representation of the general response to intravenous tPA in the Chinese population.

\section{Cluster analysis methodology}

A machine-learning method interaction tree with recursive partitioning ${ }^{19}$ was used to perform the subgroup analysis. The interaction tree model recursively partitions patients into subgroups in order to maximise the intervention effect of dosage on the risk of sICH across the subgroups. This methodology has been used in a couple of medical studies, such as in atrial fibrillation and stroke rehabilitation, ${ }^{19-21}$ but has not been applied in any acute stroke study. Based on the distribution of dosages (online supplementary figure 1) and the fact that $12 \%$ of patients were treated with lower dosages, more than $0.8 \mathrm{mg} / \mathrm{kg}$ was considered a standard dose. This cut-off dosage was selected in consideration of a conservative approach but with better reliability.

The response variable was the rate of sICH per the NINDS intracranial haemorrhage definition. The efficacy outcome was measured by an improvement in the National Institutes of Health Stroke Scale (NIHSS) score.
Such clinical improvement was defined as NIHSS 0 or 1 or an improvement of more than 4 points (within 7 days or at discharge) from admission. The following variables were included in the analysis and entered into the building of the interaction tree: demographics (sex and age), history (smoking, hypertension, diabetes, atrial fibrillation, transient ischaemic attack/stroke), clinical condition as measured by NIHSS and onset to needle time. These variables were considered as risk factors in previous sICH models. ${ }^{11-15}$

\section{Modelling to define the tree: unsupervised analysis}

The interaction tree partitions patients to optimise the heterogeneity of IV tPA effects on sICH across all patient subgroups. ${ }^{18}$ This partitioning algorithm split a parent group into two daughter groups so that the interaction effect between intravenous tPA and the daughter groups on sICH was maximised. The algorithm then took each daughter group as a parent group and repeated partitioning until the size of the daughter group reached a desired level. The analysis was conducted using the statistical software $\mathrm{R}$.

\section{Validation of the interaction model}

Bias-reduced logistic regression models for rare events ${ }^{22}$ were performed to compare the effects between lower and standard dosages within each identified patient subgroup on sICH and improvement on the secondary outcome.

\section{RESULTS}

From the pooled data of $4 \mathrm{~S}$ and TIMS-China, 3479 patients were included in this study. One in six had lower doses of intravenous tPA in the TIMS-China database and the ratio was 1 in 10 in $4 \mathrm{~S}$. The interaction tree model is demonstrated in figure 1. NIHSS score was selected by the interaction tree as the partitioning criterion, and three patient subgroups were identified: (1) mild stroke: NIHSS $\leq 4$; (2) moderate stroke: NIHSS between 5 and 14; and (3) severe stroke: NIHSS $\geq 15$.

Conventional variables used as classifiers, such as age and diabetes mellitus, did not drive the clustering. The findings were further confirmed using the bias-reduced logistic regression model for rare events (table 1). For patients with moderate severity of stroke, lower dose of intravenous tPA had less risk of sICH (OR 0.13, 95\% CI 0.02 to $0.68, \mathrm{p}=0.01$ for moderate stroke). No similar trend was seen in patients who had mild or severe stroke (OR 2.71, 95\% CI 0.80 to 7.69, $\mathrm{p}=0.10$ for mild stroke; OR $0.65,95 \%$ CI 0.19 to $2.55, \mathrm{p}=0.52$ for severe stroke). However, we noted that both the lower and standard doses had comparable efficacy for all three groups.

Furthermore, we found that the patients in the mild stroke group were younger, male predominant and had fewer vascular risk factors, while the patients in the severe stroke group had the shortest onset-to-treatment interval and the highest risk of developing sICH (table 2). Since the patients who had severe stroke were split out first, 


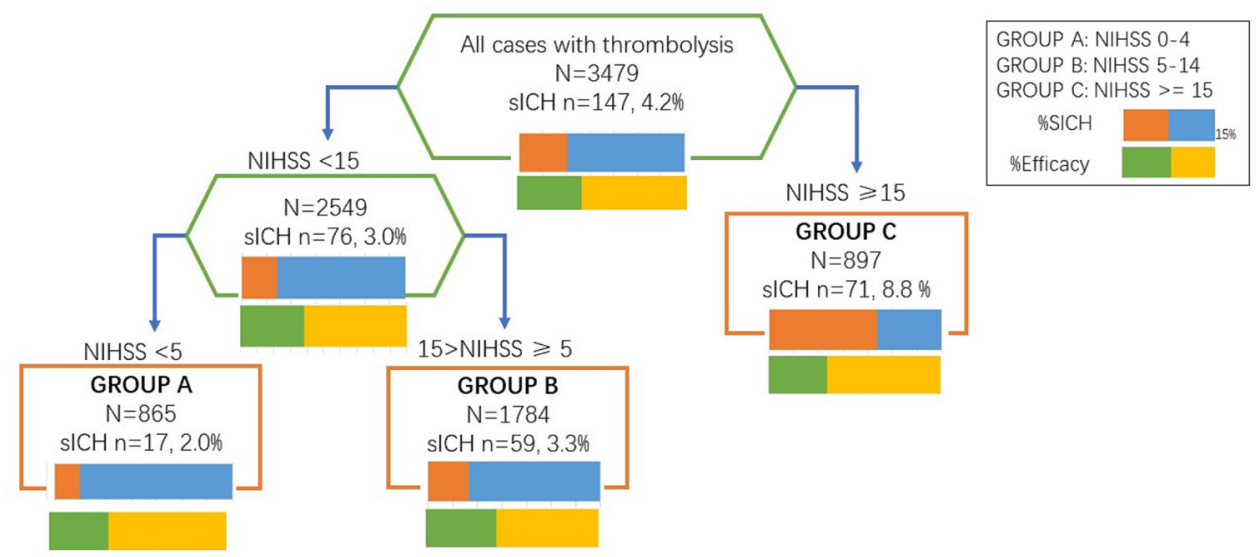

Figure 1 Interaction tree generated by supervised cluster analysis. This picture illustrates the process of interaction tree model. Group $\mathrm{C}$ was first split out, which showed the heterogeneity between group $\mathrm{C}$ and the rest in aspects of tPA effects on sICH. Then group A and group B were separated. NIHSS, National Institutes of Health Stroke Scale; sICH, symptomatic intracranial haemorrhage; tPA, tissue plasminogen activator.

they were also compared in the clinical settings (online supplementary table 3 ).

Among each severity subgroup, we analysed the rates of sICH and efficacy at different dosage levels of intravenous tPA (online supplementary table 2). Although the incidence of sICH was limited in each subset of dosages, there was a trend showing that in patients with moderate severity of stroke, the lower the dose of tPA, the less the risk of having sICH. There was no significant association between intravenous tPA dosages and risk of sICH among patients who had mild and severe strokes.

\section{DISCUSSION}

From this cluster analysis, patients with moderate severity of stroke treated with low dosage of intravenous tPA had comparable efficacy and lower risk of sICH in comparison with those treated with the standard dose. Patients who had more severe stroke were likely to have higher risk of developing sICH. Therefore, the benefit of a lower dose of intravenous tPA was only found in patients with moderate severity of stroke. No such benefit was seen when the homogeneity of intravenous tPA effects was maintained within mild and severe patient subgroups.

The safety of giving intravenous tPA to patients who had stroke has been a concern for all neurologists. Overtreatment could be costly and risky. On the other hand, undertreatment would not improve neurological deficits and cause potential severe disability. A recent editorial called for more research on the right dosage level of intravenous tPA for Asians with AIS. ${ }^{22}$ Asia now has $60 \%$ of the world's stroke victims. However, the average cost of one dose of tPA has increased $111 \%$ in one decade, from US $\$ 3050$ to US $\$ 6430$, making the acute phase treatment medical cost a financial challenge in Asia. Interestingly, the ENCHANTED trial with $63 \%$ Asian patients enrolled and which used non-inferiority design to compare low versus standard doses of intravenous tPA failed. ${ }^{10}$ The sample size of Chinese patients in ENCHANTED was relatively small to draw the conclusion. This 'Asian' tPA dosage debate is an ideal scenario where evidence-based medicine is crucial and where the medical guidelines

\begin{tabular}{|c|c|c|c|c|c|c|}
\hline & n (\%) & n1 (\%) & n2 (\%) & OR & $95 \% \mathrm{Cl}$ & $P$ value \\
\hline \multicolumn{7}{|c|}{ Interventional effect on clinical improvement } \\
\hline Mild stroke & $283(32.7)$ & $65(63.1)$ & $496(66.9)$ & 0.81 & 0.51 to 1.28 & 0.3554 \\
\hline Moderate stroke & $704(39.5)$ & $122(58.1)$ & $899(59.3)$ & 1.23 & 0.71 to 2.13 & 0.4551 \\
\hline Severe stroke & $245(30.4)$ & $59(64.8)$ & $416(66.1)$ & 1.12 & 0.58 to 2.17 & 0.7278 \\
\hline \multicolumn{7}{|c|}{ Interventional effect on sICH } \\
\hline Mild stroke & $17(1.97)$ & $4(3.77)$ & $13(1.71)$ & 2.71 & 0.80 to 7.69 & 0.1026 \\
\hline Moderate stroke & $59(3.31)$ & $2(0.94)$ & $57(3.63)$ & 0.13 & 0.02 to 0.68 & $0.0159^{*}$ \\
\hline Severe stroke & $71(8.80)$ & $14(13.3)$ & $57(8.12)$ & 0.65 & 0.19 to 2.55 & 0.5180 \\
\hline
\end{tabular}

Groups: mild: NIHSS 0-4; moderate: NIHSS 5-14; severe: NIHSS $\geq 15$.

${ }^{*} \mathrm{P}<0.05$. Compared with those treated with standard dosage, patients treated with lower dosage of tPA have significantly less risk of sICH. n1, number in low dose group; n2, number in standard dose group; $n$, total sample size in each group; NIHSS, National Institutes of Health Stroke Scale; sICH, symptomatic intracranial haemorrhage; tPA, tissue plasminogen activator. 
Table 2 Comparison of demographics and outcome among three subgroups

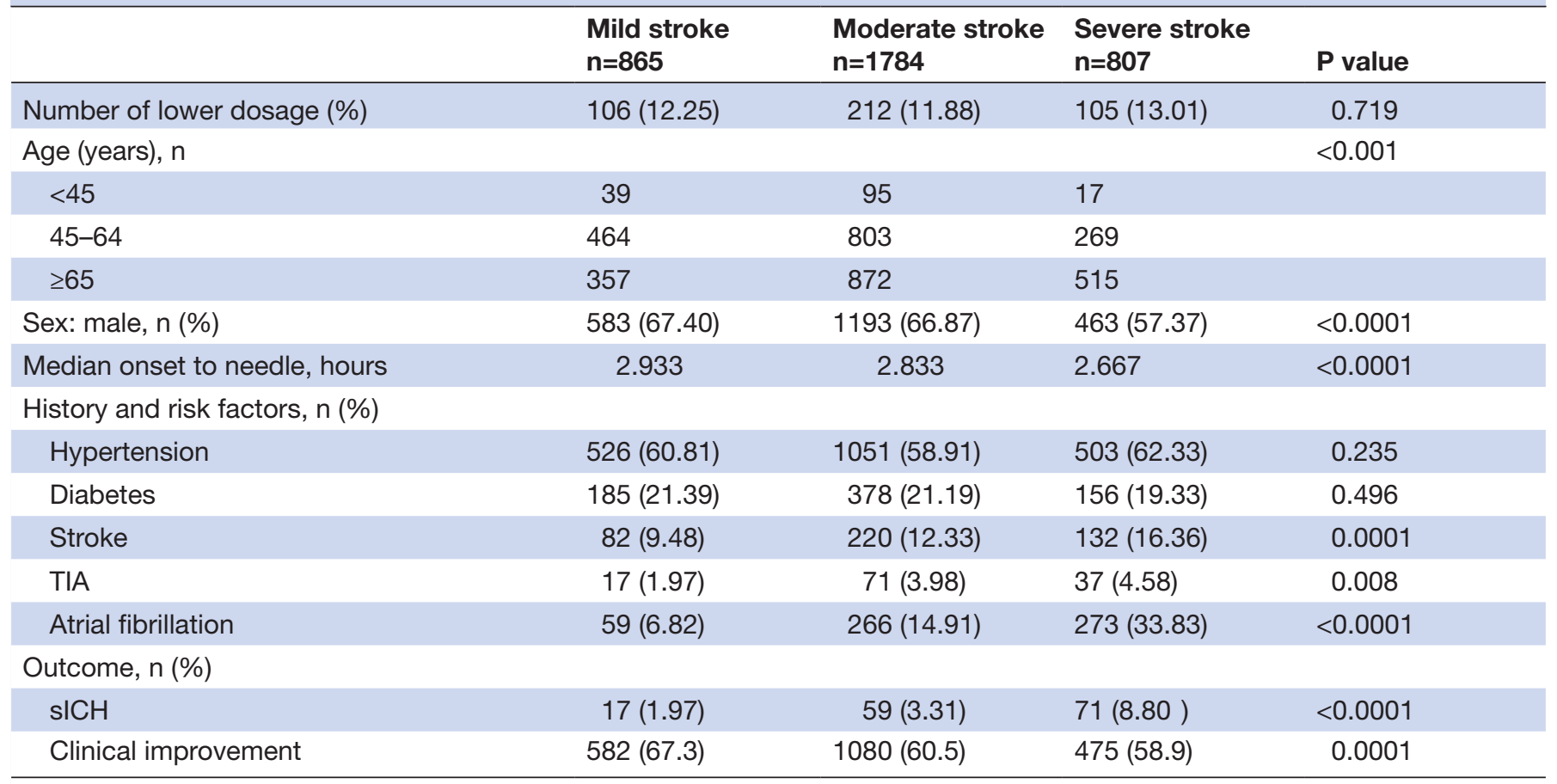

Groups: mild: NIHSS 0-4; moderate: NIHSS 5-14; severe: NIHSS $\geq 15$.

NIHSS, National Institutes of Health Stroke Scale; sICH, symptomatic intracranial haemorrhage; TIA, transient ischaemic attack.

based on studies in the West may not totally apply to Asian and Chinese patients.

In this study, cluster analysis used algorithms to group a patient population without an a priori hypothesis, which was quite different from the subgroup analyses of data from a larger trial. ${ }^{1}$ The result of our cluster analysis identified that patients with moderate severity of stroke would benefit the most from lower doses of intravenous tPA. In this subgroup, lower doses of intravenous tPA were effective as standard dose, but with a reduction in the rate of sICH.

There were several limitations to this study. The major weakness was the outcome measure. Although the modified Rankin score at 3 months is recognised as efficacious in acute stroke reperfusion, the registry lacks follow-up data. Several reperfusion studies also use improvement in NIHSS score as supportive data in early response assessment. ${ }^{23-25}$ Similarly, there were varied sICH definitions used, such as the ECASS ((European Cooperative Acute Stroke Study) and SITS-MOST(Safe Implementation of Thrombolysis in Stroke-Monitoring Study) definitions. The NINDS definition was more reliable and we only recorded NIHSS score at baseline and after tPA. In addition, the cluster analysis was also limited due to its retrospective nature, and only the variables collected at baseline were accessed. In addition, splitting into groups was automated by a computer-driven algorithm in order to maximise treatment differences. Hence, the result may not be as robust, and external validation using larger sample databases is still needed.

\section{CONCLUSION}

In conclusion, a cluster analysis of patients treated with varying dosages of intravenous tPA from two large AIS registries in China has identified the responses in three distinct groups of subjects who had a stroke based on severity. These patients responded differently to low doses of tPA versus standard dose. Patients in the moderate severity stroke cluster experienced a significantly greater reduction in the rate of sICH, yet with similar efficacy. Those in the other two groups had no additional benefit in terms of reduction in the rate of sICH. This study highlights the potential for a personalised approach to the treatment of patients with AIS. The findings also suggest that data-driven clustering methodology can be used to generate useful information to guide clinical practice and generate potential hypotheses for future studies.

\section{Author affiliations}

${ }^{1}$ Department of Neurology, Huashan Hospital Fudan University, Shanghai, Shanghai, China

${ }^{2}$ Department of Business Administration, College of Business, University of Illinois at Urbana-Champaign, Champaign, Illinois, USA

${ }^{3}$ Faculty of Business and Economics, University of Hong Kong, Hong Kong, China

${ }^{4}$ Department of Neurology, Beijing Tiantan Hospital, Capital Medical University, Beijing, China

${ }^{5}$ Medical Research Center, DotHealth, Shanghai, China

${ }^{6}$ Big data and Al dempartment, China National Clinical Research Center for Neurological Diseases, Beijing, China

Correction notice The paper has been updated since first published to update affiliations of Dr Yilong Wang, first authorship, and funding details. 
Contributors QD and YLW designed this study. YD and YH performed the analysis. HS, FM and HL supervised the analysis. YD drafted the manuscript. YJW and QD provided revisions.

Funding The Ministry of Science and Technology Major Project of China (2017YFC1310903), University of Hong Kong BRC Grant, and Patrick SC Poon Professorship Endowment Fund.

Competing interests None declared.

Patient consent for publication Not required.

Ethics approval The study is a data-driven post-hoc analysis. The original ethics approval has been approved by the Tiantan Hospital and studies have been reported. Provenance and peer review Not commissioned; externally peer reviewed. Data availability statement Data are available upon reasonable request to $Q D$ at dong_qiang@fudan.edu.cn or YLW at yilong528@aliyun.com. Data from this study for data validation and academic corporations have now been made publicly available through direct requests from both these study principal investigators.

Open access This is an open access article distributed in accordance with the Creative Commons Attribution Non Commercial (CC BY-NC 4.0) license, which permits others to distribute, remix, adapt, build upon this work non-commercially, and license their derivative works on different terms, provided the original work is properly cited, appropriate credit is given, any changes made indicated, and the use is non-commercial. See: http://creativecommons.org/licenses/by-nc/4.0/.

ORCID iDs

Yi Dong http://orcid.org/0000-0001-6630-112X

Yongjun Wang http://orcid.org/0000-0002-9976-2341

Qiang Dong http://orcid.org/0000-0002-3874-0130

\section{REFERENCES}

1 Anderson CS, Robinson T, Lindley RI, et al. Low-Dose versus standard-dose intravenous alteplase in acute ischemic stroke. $\mathrm{N} \mathrm{Engl}$ J Med 2016;374:2313-23.

2 Haley EC, Levy DE, Brott TG, et al. Urgent therapy for stroke. Part II. pilot study of tissue plasminogen activator administered 91-180 minutes from onset. Stroke 1992;23:641-5.

3 Brott TG, Haley EC, Levy DE, et al. Urgent therapy for stroke. Part I. pilot study of tissue plasminogen activator administered within 90 minutes. Stroke 1992;23:632-40.

4 The National Institute of Neurological Disorders and Stroke rt-PA Stroke Study Group. Tissue plasminogen activator for acute ischemic stroke. N Engl J Med Overseas Ed 1995;333:1581-8.

5 Chen $\mathrm{C}-\mathrm{H}$, Hsieh $\mathrm{C}-\mathrm{Y}$, Lai T-B, et al. Optimal dose for stroke thrombolysis in Asians: low dose may have similar safety and efficacy as standard dose. J Thromb Haemost 2012;10:1270-5.

6 Liao X, Wang Y, Pan Y, et al. Standard-dose intravenous tissue-type plasminogen activator for stroke is better than low doses. Stroke 2014:45:2354-8.

7 Liu M-D, Ning W-D, Wang R-C, et al. Low-dose versus standarddose tissue plasminogen activator in acute ischemic stroke in Asian populations: a meta-analysis. Medicine 2015;94:e2412.

8 Sharma VK, Tsivgoulis G, Tan JH, et al. Feasibility and safety of intravenous thrombolysis in multiethnic Asian stroke patients in Singapore. J Stroke Cerebrovasc Dis 2010;19:885-90.
9 Chao A-C, Hsu H-Y, Chung C-P, et al. Outcomes of thrombolytic therapy for acute ischemic stroke in Chinese patients: the Taiwan thrombolytic therapy for acute ischemic stroke (TTT-AIS) study. Stroke 2010;41:885-90.

10 Song L, Wang X, Robinson T, et al. Characteristics, management and response to alteplase in China versus non-China participants of the enchanted trial. Stroke Vasc Neurol 2017;2:53-8.

11 Lou M, Safdar A, Mehdiratta M, et al. The HAT score: a simple grading scale for predicting hemorrhage after thrombolysis. Neurology 2008;71:1417-23.

12 Cucchiara B, Tanne D, Levine SR, et al. A risk score to predict intracranial hemorrhage after recombinant tissue plasminogen activator for acute ischemic stroke. J Stroke Cerebrovasc Dis 2008;17:331-3.

13 Mazya M, Egido JA, Ford GA, et al. Predicting the risk of symptomatic intracerebral hemorrhage in ischemic stroke treated with intravenous alteplase: safe implementation of treatments in stroke (SITS) symptomatic intracerebral hemorrhage risk score. Stroke 2012;43:1524-31.

14 Whiteley WN, Thompson D, Murray G, et al. Targeting recombinant tissue-type plasminogen activator in acute ischemic stroke based on risk of intracranial hemorrhage or poor functional outcome: an analysis of the third International stroke trial. Stroke 2014;45:1000-6.

15 Strbian D, Engelter S, Michel P, et al. Symptomatic intracranial hemorrhage after stroke thrombolysis: the SEDAN score. Ann Neurol 2012;71:634-41.

16 Hacke W, Bluhmki E, Steiner T, et al. Dichotomized efficacy end points and global end-point analysis applied to the ECASS intention-to-treat data set: post hoc analysis of ECASS I. Stroke 1998;29:2073-5.

17 Dong Y, Fang K, Wang X, et al. The network of Shanghai stroke service system (4S): a public health-care web-based database using automatic extraction of electronic medical records. Int J Stroke 2018;13:539-44.

18 Su X, Tsai CL, Wang $\mathrm{H}$, et al. Subgroup analysis via recursive partitioning. J Mach Learn Res 2009;10:141-58.

19 Disantostefano RL, Li H, Rubin DB, et al. Which patients with chronic obstructive pulmonary disease benefit from the addition of an inhaled corticosteroid to their bronchodilator? a cluster analysis. BMJ Open 2013;3:e001838.

20 Inohara T, Shrader P, Pieper K, et al. Association of of atrial fibrillation clinical phenotypes with treatment patterns and outcomes: a multicenter registry study. JAMA Cardiol 2018;3:54-63.

21 Firth D. Bias reduction of maximum likelihood estimates. Biometrika 1993;80:27-38.

22 Sila $C$. Finding the right t-PA dose for Asians with acute ischemic stroke. N Engl J Med 2016;374:2389-90.

23 Campbell BCV, Mitchell PJ, Kleinig TJ, et al. Endovascular therapy for ischemic stroke with perfusion-imaging selection. N Engl J Med 2015;372:1009-18.

24 Nogueira RG, Jadhav AP, Haussen DC, et al. Thrombectomy 6 to 24 hours after stroke with a mismatch between deficit and infarct. $N$ Engl J Med 2018;378:11-21.

25 Yang P, Zhang Y, Zhang L, et al. Endovascular thrombectomy with or without intravenous alteplase in acute stroke. $N$ Engl J Med 2020;382:1981-93. 